\title{
Quantum-defect method and valence excitons in rare-gas solids
}

\author{
V. Saile and R. Reininger \\ Hamburger Synchrotronstrahlungslabor (HASYLAB) at DESY, D-2000 Hamburg 52, Federal Republic of Germany \\ P. Laporte \\ Equipe de Spectroscopie de Centre National de la Recherche Scientifique, F-42023 Saint-Etienne Cédex, France
}

I. T. Steinberger

Racah Institute of Physics, The Hebrew University of Jerusalem, Jerusalem, Israel

G. L. Findley

Department of Chemistry, Louisiana State University, Baton Rouge, Louisiana 70803

(Received 27 August 1987)

\begin{abstract}
In Resca's Comment on three of our papers (preceding paper), he attributes to us a much greater criticism of the quantum-defect method applied to valence excitons than we ourselves originally intended. In this Reply, we attempt to clarify the issue (i) by stating very explicitly where we differ with the original Resca-Resta quantum-defect model, and (ii) by recapitulating the experimental results which support our conclusions. In particular, we find ourselves in full agreement with the basic Resca-Resta nonstructural theory of rare-gas excitons. Unlike the original Resca-Resta quantum-defect model, however, we attribute nonatomic contributions to the exciton short-ranged potentials.
\end{abstract}

The introduction of exciton quantum defects by Resca et al. ${ }^{1,2}$ and by $\operatorname{Resta}^{3}$ was a step forward towards the development of a unified description of valence-exciton series in the rare-gas solids. These authors ${ }^{1-3}$ replaced the well-known Wannier formula ${ }^{4}$

$$
E_{n}=E_{G}-B / n^{2}
$$

where $E_{n}$ is the excitation energy of the $n$th exciton, $E_{G}$ is the band-gap energy, $B$ is the binding energy, and $n$ is the principal quantum number, by

$$
E_{n}=E_{G}-B /(n-\delta)^{2},
$$

where $\delta$ is the quantum defect.

It is unquestionable that Eq. (2) is applicable to solidrare-gas excitons. Where we differ with the comment of Resca, however, is in the interpretation of the origin of the quantum defect.

In the Resca-Resta nonstructural theory of excitons, one begins with the Schrödinger equation for the atomicrare-gas $s$ Rydberg states, namely $(\hbar=m=e=1)$,

$$
-\frac{1}{2}\left(d^{2} / d r^{2}\right) \phi_{n}^{a}(r)+U^{a}(r) \phi_{n}^{a}(r)=-T_{n}^{a} \phi_{n}^{a}(r),
$$

where $\phi_{n}^{a}(r)$ is the (reduced) radial wave function and $T_{n}^{a}$ is the term value (i.e., the difference between ionization and excitation energies) of the $n$th Rydberg state. The potential $U^{a}(r)$ has the property that

$$
U^{a}(r)= \begin{cases}-1 / r, & r>r_{0}^{a} \\ V^{a}(r), & r \leq r_{0}^{a}\end{cases}
$$

where $V^{a}(r)$ is the short-ranged residual potential which gives rise to the atomic quantum defect. For solid-rare- gas excitons, one replaces Eq. (3) by an analogous equation, namely,

$$
-\left(1 / 2 m^{*}\right)\left(d^{2} / d r^{2}\right) \phi_{n}^{s}(r)+U^{s}(r) \phi_{n}^{s}(r)=-T_{n}^{s} \phi_{n}^{s}(r),
$$

where, now, $m^{*}$ is the reduced effective mass of the exciton and $T_{n}^{s}$ is the term value (i.e., the difference between band gap and excitation energy) of the $n$th exciton. Moreover, Eq. (4) is replaced by

$$
U^{s}(r)=\left\{\begin{array}{l}
-1 / \epsilon_{0} r, \quad r>r_{0}^{s} \\
V^{s}(r), \quad r \leq r_{0}^{s}
\end{array}\right.
$$

where $\epsilon_{0}$ is the static dielectric constant of the medium and $V^{s}(r)$ is the short-ranged residual potential which gives rise to the quantum defect $\delta$ of Eq. (2).

Equations (5) and (6) are certainly applicable to solidrare-gas excitons, provided that $V^{s}(r), m^{*}$, and $\epsilon_{0}$ are chosen properly. It should be pointed out, however, that both $m^{*}$ and $\epsilon_{0}$ might have to be modified for the $n=1$ excitons with an electron-hole separation, being smaller than the nearest-neighbor distance (central-cell correction $\left.^{5,6}\right)$.

Since the Resca-Resta nonstructural theory makes no prior appeal to long-range order (i.e., band structures), and since $m^{*}$ and $\epsilon_{0}$ of Eqs. (5) and (6) are continuous functions of the rare-gas number density $\rho,{ }^{7}$ one could also hope to apply the nonstructural theory to dense rare gases and to rare-gas liquids.

The point where we differ with the Resca-Resta theory is in their additional assumption that 


$$
V^{a}(r)=V^{s}(r)
$$

In order to facilitate the calculation of quantum defects for excitons, Resca and Resta restricted Eq. (7) even more to

$$
V^{a}=V^{s}=C, \quad r_{0}^{a}=r_{0}^{s},
$$

with a constant $C$. Equations (7) and (8), of course, imply that the solid-rare-gas excitons are parentally related to the $s$ Rydberg states of the rare-gas atoms. In fact, our experimental studies and analyses, ${ }^{8-10}$ as described below, prove that Eq. (7) and consequently also Eq. (8) are invalid.

The Resca-Resta nonstructural theory, when coupled with Eq. (7), implies that the solid-rare-gas excitons evolve continuously from atomic Rydberg states. (In other words, the excitons are perturbed atomic states.) We undertook a test of this prediction by experimentally measuring the number-density dependence of the energy positions of Rydberg states and excitons in the rare gases. $^{8,11}$

For $\mathrm{Xe},{ }^{11} \mathrm{Kr}$, and $\mathrm{Ar},{ }^{8}$ the Rydberg states broaden and shift with increasing number density. At characteristic densities, new peaks appear which are distinct from the rare-gas Rydberg states, and which ultimately evolve into the solid-rare-gas excitons. (These characteristic densities are different for the different excitons.) This is illustrated in Fig. 1 for the $n=1$ exciton of $\mathrm{Kr}$. With increasing density one sees that the exciton peak grows at the expense of the Rydberg peak; moreover, the former narrows with increasing number density while the latter broadens, as is shown in Fig. 2. The most important finding, however, is that there exists an intermediate density for which both Rydberg and exciton peaks coexist. This is a clear contradiction to the Resca-Resta prediction which, when interpreted in terms of conventional line-broadening theory, ${ }^{12}$ requires a broadening of the Rydberg peak with increasing number density, leading ultimately to a tail on the exciton peak. At no intermediate

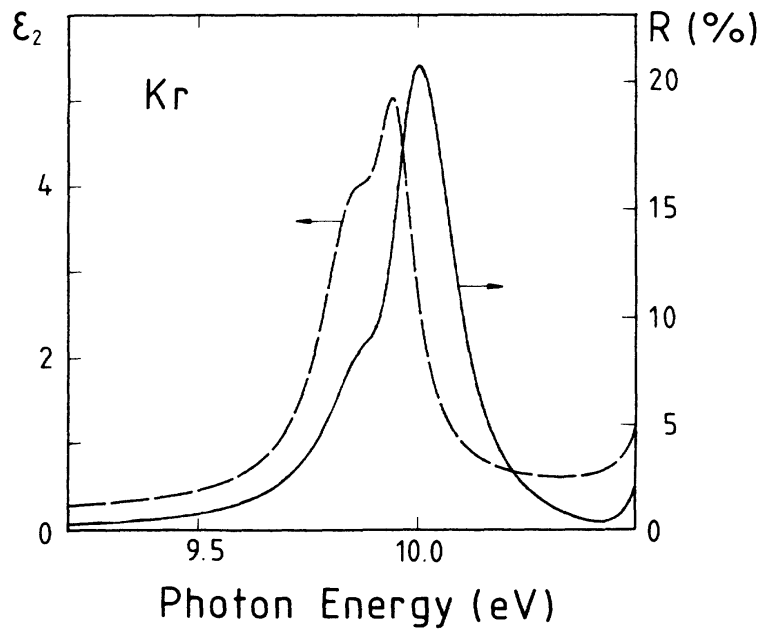

FIG. 1. Reflectivity $R$ (solid line) and imaginary part $\epsilon_{2}$ of the dielectric constant (dashed line) for the first $\mathrm{Kr}$ excitation at a number density $\rho=12.15 \times 10^{21} \mathrm{~cm}^{-3}$.

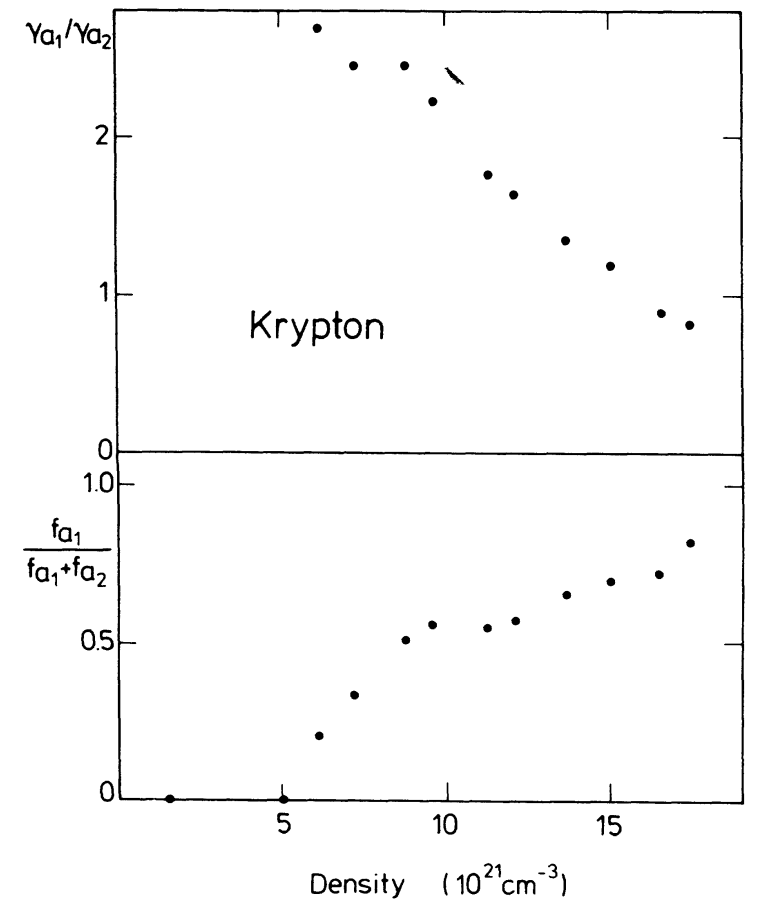

FIG. 2. Evolution of the linewidths $\gamma$ and oscillator strengths $f$ in $\mathrm{Kr}$ with number density. $\gamma_{a 1}, f_{a 1}$ correspond to the $n=1$ $\left({ }^{3} P_{1}\right)$ exciton and $\gamma_{a 2}, f_{a 2}$ to the $4 p^{6} \rightarrow 4 p^{5} 5 s^{3} P_{1}$ Rydberg transition, respectively.

density, however, should one be able to observe two separate peaks. (This contradiction remains unchanged even when one takes into account the density fluctuations mentioned in Resca's Comment. In fact, we included these fluctuations in our analysis. ${ }^{8}$ )

Thus we are forced to conclude that Eq. (7) is invalid for valence excitons in rare-gas solids. We do not thereby conclude that the nonstructural approach itself is invalid. Indeed, the appearance of excitons in dense rare gases and rare-gas liquids appears to be a striking confirmation of the nonstructural theory. The additional interactions contained within $V^{s}(r)$ might be tentatively ascribed to the transfer of excitation to neighboring atoms in a medium of sufficiently high density. At any rate, it seems clear that $V^{s}(r)$ must contain terms that are non-atomic in origin. This finding is further bolstered by densitydependent measurements of the $n=2$ exciton in $\mathrm{Xe}^{11}$ and $\mathrm{Kr}:{ }^{13}$ In this case, the exciton peak appears out of nowhere (i.e., in the absence of any nearby atom-related transition) when the dense liquid is approached.

The main result of the experimental work ${ }^{8,11}$ is that valence excitons and perturbed Rydberg states are different entities. It is worth mentioning that the latter ones in fact exhibit the expected density dependence, namely, a continuous energy shift over the entire range from the isolated atom to the solid. This can be studied most easily for doped systems, like Xe in Ar, where the peak positions of the Xe Rydberg states were monitored with increasing Ar density. ${ }^{14}$

This now brings us to the two other papers addressed in Resca's Comment, namely, Refs. 9 and 10. In Ref. 9 
we reported direct measurements of $E_{G}$ for all of the solid rare gases, and compared the experimental $E_{G}$ of $\mathrm{Ne}$ with that predicted by the Wannier formula (excluding the $n=1$ exciton), and with that predicted by the Resca-Resta nonstructural theory, assuming Eq. (7) and a constant quantum defect as originally proposed by Res$\mathrm{ta}^{3}$ Our data were of sufficiently high accuracy to distinguish between the Wannier and Resta values for $E_{G}$ in the case of $\mathrm{Ne}$. Indeed, we found that the Wannier value is more in accord with experiment than is the Resta value. Again, we suspected that Eq. (7) is invalid, at least if a constant quantum defect is assumed.

In his Comment, Resta points out that the assumption of nonconstant quantum defects leads to a prediction ${ }^{2}$ of $E_{G}$ that is in accord with our measured value, to within experimental error. We agree. However, we must point out the following.

The original calculation ${ }^{1}$ of nonconstant quantum defects assuming Eq. (7), leads to an $E_{G}$ that is not in accord with our measured value.

The improved calculation ${ }^{2}$ of nonconstant quantum defects alluded to by Resca results from an adjustment of $m^{*}$, which is not known with sufficient accuracy.

Finally, in line with the second comment above, we quote from our paper: "Certainly, the introduction of a quantum defect allows to incorporate the intermediate $n=1$ excitons in a modified Wannier formula but on the expense of an additional parameter $\delta$ which is hardly experimentally accessible if it is state dependent."

In Ref. 10 we reported empirical term-value-band-gap correlations for solid-rare-gas excitons. These correlations are linear and have the form

$$
T_{n}^{s}(M)=a_{n}^{s} E_{G}(M)+b_{n}^{s},
$$

where $M=\mathrm{Ne}, \mathrm{Ar}, \mathrm{Kr}$, and $\mathrm{Xe}$, and where the slopes and intercepts are state dependent. These correlations are analogous to those known from atomic spectroscopy, ${ }^{15}$ namely,

$$
T_{n}^{a}(M)=a_{n}^{a} I(M)+b_{n}^{a},
$$

where $I$ is an ionization limit.

In Ref. 10 we demonstrated that for both the atomic Rydberg states and the excitons the correlations [Eqs. (9) and (10)] are linear. However, we do not interpret this finding as a confirmation of the quantum-defect model.
The reason is as follows: For the atomic case the linearity between

$$
T_{n}^{a}(M)=R /[n-\delta(M)]^{2}
$$

and $I$ is due to a linear dependence of $(n-\delta)^{-2}$ on the ionization energy. The basic point is that the "unit of energy" $R$ is the same for $\mathrm{Xe}, \mathrm{Kr}, \mathrm{Ar}$, and $\mathrm{Ne}$. In the solid, however, we have to consider

$$
T_{n}^{s}(M)=B(M) /[n-\delta(M)]^{2},
$$

with $B(M)$ varying strongly between $\approx 1 \mathrm{eV}$ for $\mathrm{Xe}$ to $\approx 4-5 \mathrm{eV}$ for $\mathrm{Ne}$. From the correlation plots, especially for excitons with $n \geq 2$, we can easily conclude, that $B(M)$ rather than $[n-\delta(M)]^{-2}$ varies linearly with $E_{G}(M)$. Furthermore, rescaling of the solid correlation plot by

$$
B=\left(m^{*} / \epsilon_{0}^{2}\right) R
$$

does not yield the atomic correlation plot, as was pointed out in Ref. 10. This means, that even after correcting for the most obvious solid-state effects, we are not able to reveal an atomic parentage of the excitons in the correlation plots.

In summary, we continue to stand by the statement of Saile and Koch, ${ }^{16}$ namely, that the Resca-Resta nonstructural theory of valence excitons in the solid rare gases was a "remarkable progress in describing the whole exciton series for all rare-gas solids." We accept the validity of Eq. (5) for solid-rare-gas excitons (provided $m^{*}$ and $\epsilon_{0}$ are carefully evaluated for small excitons). Moreover, we accept the validity of Eq. (2), since it is directly implied by Eq. (5). For the evaluation of experimental data, however, Eq. (2) is rather useless, if the quantum defects $\delta$ (and probably even the binding energies $B$ for the $n=1$ excitons) are state dependent. Where we disagree with the Resca-Resta theory is in the choice of the short-ranged potential $V^{s}(r)$. We persist in attributing nonatomic terms to $V^{s}(r)$, and consequently nonatomic contributions to the quantum defects resulting from $V^{s}(r)$.

G. L. F. acknowledges support from the U.S. Department of Energy.
${ }^{1}$ L. Resca, R. Resta, and S. Rodriguez, Phys. Rev. B 18, 696 (1978).

${ }^{2}$ L. Resca and R. Resta, Phys. Rev. B 19, 1683 (1979).

${ }^{3}$ R. Resta, Phys. Status Solidi B 86, 627 (1978).

${ }^{4}$ G. H. Wannier, Phys. Rev. 52, 191 (1937).

${ }^{5}$ J. Hermanson and J. C. Phillips, Phys. Rev. 150, 652 (1966).

6J. Hermanson, Phys. Rev. 150, 660 (1966).

${ }^{7}$ R. Reininger, U. Asaf, I. T. Steinberger, and S. Basak, Phys. Rev. B 28, 4426 (1983); R. Reininger, U. Asaf, I. T. Steinberger, V. Saile, and P. Laporte, ibid. 28, 3193 (1983).
${ }^{8}$ P. Laporte, J. L. Subtil, R. Reininger, V. Saile, S. Bernstorff, and I. T. Steinberger, Phys. Rev. B 35, 6270 (1987).

${ }^{9}$ S. Bernstorff and V. Saile, Opt. Commun. 58, 181 (1986).

${ }^{10} \mathrm{~S}$. Bernstorff, V. Saile, and G. L. Findley, Chem. Phys. Lett. 125, 161 (1986).

${ }^{11}$ P. Laporte, J. L. Subtil, U. Asaf, I. T. Steinberger, and S. Wind, Phys. Rev. Lett. 45, 2138 (1980).

${ }^{12}$ See, for example, N. Allard and J. Kielkopf, Rev. Mod. Phys. 54, 1103 (1982).

${ }^{13}$ P. Laporte, J. L. Subtil, R. Reininger, V. Saile, and I. T. Stein- 
berger, Chem. Phys. Lett. 122, 525 (1985).

${ }^{14} \mathrm{R}$. Reininger and V. Saile, 11th Molecular Crystal Symposium, Lugano, Switzerland, 1985, Extended Abstracts, p. 249 (unpublished); R. Reininger, V. Saile, and P. Laporte (unpub- lished).

${ }^{15}$ S. Chattopadhyay, P. Hochmann, and S. P. McGlynn, J. Chem. Phys. 65, 3341 (1976).

${ }^{16}$ V. Saile and E. E. Koch, Phys. Rev. B 20, 784 (1979). 